Homology, Homotopy and Applications, vol.10(2), 2008, pp.149-174

\title{
ADDING INVERSES TO DIAGRAMS ENCODING ALGEBRAIC STRUCTURES
}

\author{
JULIA E. BERGNER
}

(communicated by J. F. Jardine)

\begin{abstract}
We modify a previous result, which showed that certain diagrams of spaces are essentially simplicial monoids, to construct diagrams of spaces which model simplicial groups. Furthermore, we show that these diagrams can be generalized to models for Segal groupoids. We then modify Segal's model for simplicial abelian monoids in such a way that it becomes a model for simplicial abelian groups.
\end{abstract}

\section{Introduction}

Much research has been done on various structures equivalent to topological or simplicial groups. Classical examples include Thomason's work with delooping machines $[\mathbf{2 7}]$ and Stasheff's group-like $A_{\infty}$-spaces [26], while more modern examples make use of the structure of algebraic theories theories $[\mathbf{1}, \mathbf{2}]$. In $[\mathbf{7}]$, we focus instead on simplicial monoids. In particular, we consider diagrams of spaces which essentially give one of the spaces the structure of a monoid. While such a structure is given when the diagram is the theory of monoids, we obtain a simpler diagram which, from the viewpoint of homotopy theory, encodes the same structure.

Here we return to the original situation and ask if this construction can be modified to obtain a diagram encoding the structure of a simplicial group rather than a simplicial monoid. After all, we need only to find a way to include inverses. In this paper, we show that indeed we can represent simplicial groups in this manner.

Furthermore, as the construction for simplicial monoids generalizes to the manyobject case of simplicial categories, we can obtain models for simplicial groupoids as well. In doing so, we extend a result relating simplicial categories to Segal categories (essentially simplicial categories with composition only given up to higher homotopy) to one relating simplicial groupoids to Segal groupoids.

One might ask, then, if there are other algebraic structures which can be modelled in a similar way, in particular by some kind of diagram which is simpler than the one given by the corresponding algebraic theory. An answer for the case of simplicial abelian monoids is given by Segal's category $\Gamma[\mathbf{2 4}]$, although it does not provide the relationship between strict and homotopy structures that we have in the non-abelian situation. Here, we extend this construction to find a model for abelian groups.

Received October 9, 2006, revised June 10, 2008; published on October 22, 2008.

2000 Mathematics Subject Classification: 55U10, 18B40, 18C10, 55P35.

Key words and phrases: simplicial groups, Segal groupoids, diagram categories.

This article is available at http://intlpress.com/HHA/v10/n2/a8

Copyright (C) 2008, International Press. Permission to copy for private use granted. 
To obtain this result, we use work of Bousfield, in which he modifies the projections in the diagram for simplicial monoids, rather than the diagram itself, to encode inverses and so model simplicial groups [11]. To model simplicial abelian groups, we change the projections in the category $\Gamma$ encoding an abelian monoid structure in an analogous way. It should be noted that Bousfield's work includes models for $n$-fold loop spaces, work which has been approached from another angle by Berger [4].

Of course, the question we answer here in these cases can be asked for many other algebraic structures. For example, are there simple diagrams encoding the structure of (commutative) rings? We can also ask about so-called multi-sorted algebraic structures, such as operads and group actions, for which we have models given by multisorted algebraic theories $[6]$. We hope to find more such results in future work. In

related work, Barwick has found diagrams given by operator categories which model certain kinds of algebraic structures $[\mathbf{3}]$.

In Sections 2 and 3, we review basic properties of model categories and some of the model category structures that we use in this paper. In Section 4, we modify the argument given in [7] for simplicial monoids to obtain a model for simplicial groups. We extend this argument in Section 5 to simplicial groupoids with a fixed object set. In Section 6, we summarize the construction of Bousfield's alternative model for simplicial groups. Finally, in Section 7, we summarize Segal's argument for a model for simplicial abelian monoids and modify it to obtain a model for simplicial abelian groups.

\section{Acknowledgements}

I would like to thank Bernard Badzioch and Bill Dwyer for their helpful suggestions for this paper, particularly with regard to the approach to simplicial abelian groups, and Clark Barwick and Joachim Kock for conversations about the category $\mathbf{I} \boldsymbol{\Delta}^{o p}$. I am also grateful to the referee for helpful comments and corrections.

\section{Model category structures}

In this section, we review the necessary tools from model categories that we will need to prove our result for simplicial groups.

Recall that a model category structure on a category $\mathcal{C}$ is a choice of three distinguished classes of morphisms: fibrations, cofibrations, and weak equivalences. A (co)fibration which is also a weak equivalence will be called an acyclic (co)fibration. With this choice of three classes of morphisms, $\mathcal{C}$ is required to satisfy five axioms MC1-MC5 $[\mathbf{1 3}, 3.3]$. Here we only state MC4 and MC5, as they are used in the course of the paper.

- (MC4) If $i: A \rightarrow B$ is a cofibration and $p: X \rightarrow Y$ is a fibration, then a dotted arrow lift exists in any solid arrow diagram of the form

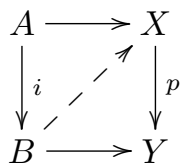


if either

1. $p$ is a weak equivalence, or

2. $i$ is a weak equivalence.

(In this case we say that $i$ has the left lifting property with respect to $p$ and that $p$ has the right lifting property with respect to $i$.)

- (MC5) Any map $f$ can be factored two ways:

1. $f=p i$, where $i$ is a cofibration and $p$ is an acyclic cofibration, and

2. $f=q j$, where $j$ is an acyclic cofibration and $q$ is a fibration.

An object $X$ in $\mathcal{C}$ is fibrant if the unique map $X \rightarrow *$ from $X$ to the terminal object is a fibration. Dually, $X$ is cofibrant if the unique map $\phi \rightarrow X$ from the initial object to $X$ is a cofibration. The factorization axiom MC5 guarantees that each object $X$ has a weakly equivalent fibrant replacement $\widehat{X}$ and a weakly equivalent cofibrant replacement $\widetilde{X}$. These replacements are not necessarily unique, but they can be chosen to be functorial in the cases we will use $[\mathbf{1 7}, 1.1 .3]$.

The model category structures which we will discuss are all cofibrantly generated. In a cofibrantly generated model category, there are two sets of morphisms, one of generating cofibrations and one of generating acyclic cofibrations, such that a map is a fibration if and only if it has the right lifting property with respect to the generating acyclic cofibrations, and a map is an acyclic fibration if and only if it has the right lifting property with respect to the generating cofibrations [15, 11.1.2]. The following theorem is useful for proving the existence of a cofibrantly generated model structure on a category.

Theorem 2.1 ([15, 11.3.1]). Let $\mathcal{M}$ be a category which has all small limits and colimits. Suppose that $\mathcal{M}$ has a class of weak equivalences which satisfies the two-outof-three property (model category axiom MC2) and which is closed under retracts. Let $I$ and $J$ be sets of maps in $\mathcal{M}$ which satisfy the following conditions:

1. Both $I$ and $J$ permit the small object argument [15, 10.5.15].

2. Every $J$-cofibration is an I-cofibration and a weak equivalence.

3. Every I-injective is a J-injective and a weak equivalence.

4. One of the following conditions holds:

(a) A map that is an I-cofibration and a weak equivalence is a J-cofibration, or

(b) A map that is both a J-injective and a weak equivalence is an I-injective.

Then there is a cofibrantly generated model category structure on $\mathcal{M}$ in which $I$ is a set of generating cofibrations and $J$ is a set of generating acyclic cofibrations.

We now state the definition of a Quillen pair of model category structures. Recall that for categories $\mathcal{C}$ and $\mathcal{D}$ a pair of functors

$$
F: \mathcal{C} \rightleftarrows \mathcal{D}: R
$$

is an adjoint pair if for each object $X$ of $\mathcal{C}$ and object $Y$ of $\mathcal{D}$ there is a natural isomorphism $\psi: \operatorname{Hom}_{\mathcal{D}}(F X, Y) \rightarrow \operatorname{Hom}_{\mathcal{C}}(X, R Y)[\mathbf{1 9}$, IV.1]. The adjoint pair is sometimes written as the triple $(F, R, \psi)$. 
Definition $2.2([\mathbf{1 7}, 1.3 .1])$. If $\mathcal{C}$ and $\mathcal{D}$ are model categories, then an adjoint pair $(F, R, \psi)$ between them is a Quillen pair if one of the following equivalent statements holds:

1. F preserves cofibrations and acyclic cofibrations.

2. $R$ preserves fibrations and acyclic fibrations.

We now have the following definition of Quillen equivalence, which is the standard notion of equivalence of model category structures.

Definition 2.3 ([17, 1.3.12]). A Quillen pair is a Quillen equivalence if for all cofibrant $X$ in $\mathcal{C}$ and fibrant $Y$ in $\mathcal{D}$, a map $f: F X \rightarrow Y$ is a weak equivalence in $\mathcal{D}$ if and only if the map $\psi f: X \rightarrow R Y$ is a weak equivalence in $\mathcal{C}$.

We will use the following proposition to prove that our Quillen pairs are Quillen equivalences. Recall that a functor $F: \mathcal{C} \rightarrow \mathcal{D}$ reflects a property if, for any morphism $f$ of $\mathcal{C}$, whenever $F f$ has the property, then so does $f$.

Proposition 2.4 ([17, 1.3.16]). Suppose that $(F, R, \psi)$ is a Quillen pair from $\mathcal{C}$ to $\mathcal{D}$. Then the following statements are equivalent:

1. $(F, R, \psi)$ is a Quillen equivalence.

2. $R$ reflects weak equivalences between fibrant objects, and for every cofibrant $X$ in $\mathcal{C}$ the map $X \rightarrow R(F X)^{f}$ is a weak equivalence.

Throughout this paper, we use the category of simplicial sets, denoted $\mathcal{S} S$ ets. Recall that a simplicial set is a functor $\boldsymbol{\Delta}^{o p} \rightarrow \mathcal{S}$ ets, where $\boldsymbol{\Delta}$ denotes the cosimplicial category whose objects are the finite ordered sets $[n]=(0 \rightarrow \cdots \rightarrow n)$ and whose morphisms are the order-preserving maps. The simplicial category $\boldsymbol{\Delta}^{o p}$ is then the opposite of this category. Some examples of simplicial sets are, for each $n \geqslant 0$, the $n$-simplex $\Delta[n]$, its boundary $\dot{\Delta}[n]$, and, for any $0 \leqslant k \leqslant n$, the simplicial set $V[n, k]$, which is $\dot{\Delta}[n]$ with the $k$ th face removed [14, I.1]. More generally, a simplicial object in a category $\mathcal{C}$ is a functor $\boldsymbol{\Delta}^{o p} \rightarrow \mathcal{C}$. In particular, a functor $\boldsymbol{\Delta}^{o p} \rightarrow \mathcal{S} \mathcal{S}$ ets is a simplicial space or bisimplicial set $[\mathbf{1 4}, \mathrm{IV}]$.

In a slight abuse of terminology, we use the term simplicial category to refer to a simplicial object in the category of all (small) categories which satisfies the additional condition that the face and degeneracy maps are the identity on all the objects. Such an object is often called a category enriched over simplicial sets, since it is just a category with a simplicial set of morphisms between any two objects. For any objects $X$ and $Y$ in a simplicial category, we denote this simplicial set $\operatorname{Map}(X, Y)$ and call it a function complex. A simplicial category in this sense with all morphisms invertible is called a simplicial groupoid.

We also use the notion of a simplicial model category $\mathcal{M}$, or a model category which is also a simplicial category satisfying two axioms [15, 9.1.6]. It is important to note that a function complex in a simplicial model category is only homotopy invariant in the case that $X$ is cofibrant and $Y$ is fibrant. For the general case, we have the following definition:

Definition 2.5 ([15, 17.3.1]). A homotopy function complex $\operatorname{Map}^{h}(X, Y)$ in a simplicial model category $\mathcal{M}$ is the simplicial set $\operatorname{Map}(\widetilde{X}, \widehat{Y})$, where $\widetilde{X}$ is a cofibrant replacement of $X$ in $\mathcal{M}$ and $\widehat{Y}$ is a fibrant replacement for $Y$. 
Several of the model category structures that we use are obtained by localizing a given model category structure with respect to a map or a set of maps. Suppose that $P=\{f: A \rightarrow B\}$ is a set of maps with respect to which we would like to localize a model category $\mathcal{M}$.

Definition 2.6. A $P$-local object $W$ is a fibrant object of $\mathcal{M}$ such that for any $f: A \rightarrow B$ in $P$, the induced map on homotopy function complexes

$$
f^{*}: \operatorname{Map}^{h}(B, W) \rightarrow \operatorname{Map}^{h}(A, W)
$$

is a weak equivalence of simplicial sets. A map $g: X \rightarrow Y$ in $\mathcal{M}$ is then a $P$-local equivalence if for every local object $W$, the induced map on homotopy function complexes

$$
g^{*}: \operatorname{Map}^{h}(Y, W) \rightarrow \operatorname{Map}^{h}(X, W)
$$

is a weak equivalence of simplicial sets.

Theorem 2.7 ([15, 4.1.1]). Let $\mathcal{M}$ be a left proper cellular model category [15, 13.1.1, 12.1.1] and $P$ a set of morphisms of $\mathcal{M}$. There is a model category structure $\mathcal{L}_{P} \mathcal{M}$ on the underlying category of $\mathcal{M}$ such that:

1. The weak equivalences are the $P$-local equivalences.

2. The cofibrations are precisely the cofibrations of $\mathcal{M}$.

3. The fibrations are the maps which have the right lifting property with respect to the maps which are both cofibrations and P-local equivalences.

4. The fibrant objects are the P-local objects.

In this situation, we refer to the functorial fibrant replacement functor as a localization functor.

When we are working with localized model category structures, the following theorem can be used to prove that an adjoint pair is still a Quillen pair after localization.

Theorem 2.8 ([15, 3.3.20]). Let $\mathcal{C}$ and $\mathcal{D}$ be left proper, cellular model categories and let $(F, R, \psi)$ be a Quillen pair between them. Let $S$ be a set of maps in $\mathcal{C}$ and $L_{S} \mathcal{C}$ the localization of $\mathcal{C}$ with respect to $S$. Then if $\mathbf{L} F S$ is the set in $\mathcal{D}$ obtained by applying the left derived functor of $F$ to the set $S[15,8.5 .11]$, then $(F, R, \psi)$ is also a Quillen pair between the model categories $L_{S} \mathcal{C}$ and $L_{\mathbf{L} F S} D$.

One important model category structure we will use is the standard one on the category $\mathcal{S}$ Sets of simplicial sets. In this case, a weak equivalence is a map of simplicial sets $f: X \rightarrow Y$ such that the induced map $|f|:|X| \rightarrow|Y|$ is a weak homotopy equivalence of topological spaces. The cofibrations are monomorphisms, and the fibrations are the maps with the right lifting property with respect to the acyclic cofibrations [14, I.11.3]. This model category structure is cofibrantly generated; a set of generating cofibrations is $I=\{\dot{\Delta}[n] \rightarrow \Delta[n] \mid n \geqslant 0\}$, and a set of generating acyclic cofibrations is $J=\{V[n, k] \rightarrow \Delta[n] \mid n \geqslant 1,0 \leqslant k \leqslant n\}$.

\section{Model categories of diagrams}

The objects of all the categories we consider in this paper are given by diagrams of spaces. Given any small category $\mathcal{D}$, there is a category $\mathcal{S} \operatorname{Sets}^{\mathcal{D}}$ of $\mathcal{D}$-diagrams 
in $\mathcal{S}$ Sets, or functors $\mathcal{D} \rightarrow \mathcal{S} \mathcal{S}$ ets. We can obtain two model category structures on $\mathcal{S} \mathcal{S e t s}^{\mathcal{D}}$ by the following results.

Theorem 3.1 ([14, IX 1.4]). Given the category $\mathcal{S} \mathcal{S}$ ets ${ }^{\mathcal{D}}$ of $\mathcal{D}$-diagrams of simplicial sets, there is a simplicial model category structure $\mathcal{S} \mathcal{S}$ ets $s_{f}^{\mathcal{D}}$ in which the weak equivalences and fibrations are objectwise and in which the cofibrations are the maps which have the left lifting property with respect to the maps which are both fibrations and weak equivalences.

Theorem $3.2\left(\left[14\right.\right.$, VIII 2.4]). There is a simplicial model category $\mathcal{S} \mathcal{S} e t s_{c}^{\mathcal{D}}$ in which the weak equivalences and the cofibrations are objectwise and in which the fibrations are the maps which have the right lifting property with respect to the maps which are cofibrations and weak equivalences.

Given these general results, we now turn to the particular diagrams which we will be considering in this paper. Let $G$ be a simplicial group, by which we mean a simplicial object in the category $\mathcal{G} r p$ of groups, or a functor $\boldsymbol{\Delta}^{o p} \rightarrow \mathcal{G r p}$. However, here we use an alternate viewpoint in which we use algebraic theories to define simplicial groups. We begin with the definition of an algebraic theory. Some references for algebraic theories include Chapter 3 of [10], the introduction to [1] , and Section 3 of $[6]$.

Definition 3.3. An algebraic theory $\mathcal{T}$ is a small category with finite products and objects denoted $T_{n}$ for $n \geqslant 0$. For each $n, T_{n}$ is equipped with an isomorphism $T_{n} \cong\left(T_{1}\right)^{n}$. Note, in particular, that $T_{0}$ is the terminal object in $T$.

Here we consider one particular theory, the theory of groups, which we denote $\mathcal{T}_{G}$. To describe this theory, we first consider the full subcategory of the category of groups generated by representatives $T_{n}$ of the isomorphism classes of free groups on $n$ generators. We then define the theory of groups $\mathcal{T}_{G}$ to be the opposite of this category. Thus $T_{n}$, which is canonically the coproduct of $n$ copies of $T_{1}$ in the category $\mathcal{G r p}$ of groups, becomes the product of $n$ copies of $T_{1}$ in $\mathcal{T}_{G}$. It follows that there is a projection map $p_{n, i}: T_{n} \rightarrow T_{1}$ for each $1 \leqslant i \leqslant n$ in addition to other group homomorphisms. In fact, there are such projection maps in any algebraic theory. We use them to make the following definition.

Definition $3.4([\mathbf{1}, 1.1])$. Given an algebraic theory $\mathcal{T}$, a strict simplicial $\mathcal{T}$-algebra (or simply $\mathcal{T}$-algebra) $A$ is a product-preserving functor $A: \mathcal{T} \rightarrow \mathcal{S} \mathcal{S} e t s$. Here, "product-preserving" means that for each $n \geqslant 0$ the canonical map

$$
A\left(T_{n}\right) \rightarrow A\left(T_{1}\right)^{n},
$$

induced by the $n$ projection maps $T_{n} \rightarrow T_{1}$, is an isomorphism of simplicial sets. In particular, $A\left(T_{0}\right)$ is the one-point simplicial set $\Delta[0]$.

In general, a $\mathcal{T}$-algebra $A$ defines a strict algebraic structure on the space $A\left(T_{1}\right)$ corresponding to the theory $\mathcal{T}[\mathbf{1}, \S 1]$. So, a $\mathcal{T}_{G}$-algebra $A$ defines a group structure on the space $A\left(T_{1}\right)$. In fact, the category of simplicial groups is equivalent to the category of $\mathcal{T}_{G}$-algebras $[\mathbf{1 8}],[\mathbf{2 0}$, II.4].

We can also consider the case where the products are not preserved strictly, but only up to homotopy. 
Definition 3.5 $([\mathbf{1}, 1.2])$. Given an algebraic theory $\mathcal{T}$, a homotopy $\mathcal{T}$-algebra is a functor $X: \mathcal{T} \rightarrow \mathcal{S}$ Sets which preserves products up to homotopy. The functor $X$ preserves products up to homotopy if, for each $n \geqslant 0$ the canonical map

$$
X\left(T_{n}\right) \rightarrow X\left(T_{1}\right)^{n}
$$

induced by the projection maps $p_{n, i}: T_{n} \rightarrow T_{1}$ for $1 \leqslant i \leqslant n$ is a weak equivalence of simplicial sets. In particular, we assume that $X\left(T_{0}\right)$ is weakly equivalent to $\Delta[0]$.

We now consider the corresponding model category structures.

Proposition 3.6 ([20, II.4], $[\mathbf{2 3}, 3.1])$. Let $\mathcal{T}$ be an algebraic theory and $\mathcal{A l g}{ }^{\mathcal{T}}$ the category of $\mathcal{T}$-algebras. Then there is a cofibrantly generated model category structure on $\mathcal{A l g}^{\mathcal{T}}$ in which the weak equivalences and fibrations are levelwise weak equivalences of simplicial sets, and the cofibrations are the maps with the left lifting property with respect to the maps which are fibrations and weak equivalences.

We also need a model category structure for homotopy $\mathcal{T}$-algebras. However, there is no model category structure on the category of homotopy $\mathcal{T}$-algebras itself since this category does not have all small colimits. However, there is a model category structure on the category of all $\mathcal{T}$-diagrams of simplicial sets in which the fibrant objects are homotopy $\mathcal{T}$-algebras. To obtain this structure, we begin by considering the model category structure $\mathcal{S} \mathcal{S}$ ets $s_{f}^{\mathcal{T}}$ on the category of all functors $\mathcal{T} \rightarrow \mathcal{S} \mathcal{S} e t s$. The desired model structure can be obtained by localizing the model structure $\mathcal{S} \mathcal{S} e t s_{f}^{\mathcal{T}}$ with respect to a set of maps. We summarize this localization here; a complete description is given by Badzioch $[\mathbf{1}, \S 5]$.

Given an algebraic theory $\mathcal{T}$, consider the functor $\operatorname{Hom}_{\mathcal{T}}\left(T_{n},-\right)$. We then have maps

$$
p_{n}: \coprod_{i=1}^{n} \operatorname{Hom}_{\mathcal{T}}\left(T_{1},-\right) \rightarrow \operatorname{Hom}_{\mathcal{T}}\left(T_{n},-\right)
$$

induced from the projection maps in $\mathcal{T}$. We then localize the model category structure on $\mathcal{S} \mathcal{S}$ ets ${ }^{\mathcal{T}}$ with respect to the set $S=\left\{p_{n} \mid n \geqslant 0\right\}$. We denote the resulting model category structure $\mathcal{L} \mathcal{S} \mathcal{S e t s}^{\mathcal{T}}$.

Proposition 3.7 ([1, 5.5]). The fibrant objects in $\mathcal{L} \mathcal{S} \mathcal{S e t s}^{\mathcal{T}}$ are the homotopy $\mathcal{T}$-algebras which are fibrant in $\mathcal{S} \mathcal{S e t s}^{\mathcal{T}}$.

We now have the following result by Badzioch which relates strict and homotopy $\mathcal{T}$-algebras.

Theorem $3.8([\mathbf{1}, 6.4])$. Given an algebraic theory $\mathcal{T}$, there is a Quillen equivalence of model categories between $\mathcal{A l g}^{\mathcal{T}}$ and $\mathcal{L} \mathcal{S} S e t s^{\mathcal{T}}$.

We will find it convenient, however, to work in the situation where a homotopy $\mathcal{T}$-algebra $X$ has $X_{0}$ precisely $\Delta[0]$ rather than just a space weakly equivalent to it. The following two results are proved in $[\mathbf{7}]$ and $[\mathbf{8}]$ for the theory of monoids $\mathcal{T}_{M}$, but their proofs hold for any algebraic theory $\mathcal{T}$. 
Proposition 3.9 ([7,3.11]). Consider the category $\mathcal{S} \mathcal{S}$ ets ${ }_{*}^{\mathcal{T}}$ of functors $\mathcal{T} \rightarrow \mathcal{S}$ Sets such that the image of $T_{0}$ is $\Delta[0]$. There is a model category structure on $\mathcal{S} \mathcal{S} e t s_{*}^{\mathcal{T}}$ in which the weak equivalences and fibrations are defined levelwise and the cofibrations are the maps with the left lifting property with respect to the acyclic fibrations.

Now, to obtain a localized model category $\mathcal{L S S}$ et $s_{*}^{\mathcal{T}}$, we need to modify the maps

$$
p_{n}: \coprod_{i=1}^{n} \operatorname{Hom}_{\mathcal{T}}\left(T_{1},-\right) \rightarrow \operatorname{Hom}_{\mathcal{T}}\left(T_{n},-\right)
$$

that we used to obtain $\mathcal{L S S}$ ets ${ }^{\mathcal{T}}$ from $\mathcal{S} \mathcal{S} e t s^{\mathcal{T}}$. Since $\operatorname{Hom}_{\mathcal{T}}\left(T_{n},-\right)_{0} \cong \Delta[0]$ for all $n \geqslant 0$, the only change we need to make to these maps is to take the coproduct $\coprod_{n} \operatorname{Hom}_{\mathcal{T}}\left(T_{1},-\right)$ in the category $\mathcal{S} \mathcal{S} e t s_{*}^{\mathcal{T}_{M}}$ (as in $\left.[\mathbf{7}, 3.6]\right)$. We then localize $\mathcal{S} \mathcal{S} e t s_{*}^{\mathcal{T}_{M}}$ with respect to the set of all such maps to obtain a model structure $\mathcal{L S} \mathcal{S}$ et $s_{*}^{\mathcal{T}}$.

Since a fibrant and cofibrant object $X$ in $\mathcal{L} \mathcal{S} \mathcal{S e t}{ }^{\mathcal{T}}$ has $X_{0}$ weakly equivalent to $\Delta[0]$, it is not too surprising that we can instead use $\mathcal{L S S} \mathcal{S e t} s_{*}^{\mathcal{T}}$ in the following variation on Badzioch's result:

Proposition $3.10([8])$. There is a Quillen equivalence of model categories

$$
L: \mathcal{L S S e t s} s_{*}^{\mathcal{T}} \longleftrightarrow \mathcal{A l g}{ }^{\mathcal{T}}: I,
$$

where $I$ denotes the inclusion functor and $L$ is its left adjoint.

Now that we have established our definitions for simplicial groups, we turn to reduced Segal categories and reduced Segal groupoids. These are simplicial spaces satisfying some conditions, so, like the simplicial monoids, they are given by a diagram of simplicial sets. We begin with the definition of a Segal precategory.

Definition 3.11. A Segal precategory is a simplicial space $X$ such that $X_{0}$ is a discrete simplicial set. If $X_{0}$ consists of a single point, then $X$ is a reduced Segal precategory.

Now note that for any simplicial space $X$ there is a Segal map

$$
\varphi_{n}: X_{n} \rightarrow \underbrace{X_{1} \times_{X_{0}} \ldots \times_{X_{0}} X_{1}}_{n}
$$

for each $n \geqslant 2$, which we define as follows. Let $\alpha^{k}:[1] \rightarrow[n]$ be the map in $\boldsymbol{\Delta}$ such that $\alpha^{k}(0)=k$ and $\alpha^{k}(1)=k+1$, defined for each $0 \leqslant k \leqslant n-1$. We can then define the dual maps $\alpha_{k}:[n] \rightarrow[1]$ in $\boldsymbol{\Delta}^{o p}$. For $n \geqslant 2$, the Segal map is defined to be the map

$$
\varphi_{n}: X_{n} \rightarrow \underbrace{X_{1} \times_{X_{0}} \cdots \times_{X_{0}} X_{1}}_{n}
$$

induced by the maps

$$
X\left(\alpha_{k}\right): X_{n} \rightarrow X_{1}
$$


Definition $3.12([\mathbf{1 6}, \S 2])$. A Segal category $X$ is a Segal precategory $X: \boldsymbol{\Delta}^{o p} \rightarrow$ $\mathcal{S} S$ ets such that $X_{0}=\Delta[0]$ and such that for each $n \geqslant 2$ the Segal map

$$
\varphi_{n}: X_{n} \rightarrow \underbrace{X_{1} \times_{X_{0}} \cdots \times_{X_{0}} X_{1}}_{n}
$$

is a weak equivalence of simplicial sets. If $X_{0}=\Delta[0]$, then $X$ is a reduced Segal category. Note that in this case we have $X_{n} \simeq\left(X_{1}\right)^{n}$.

We now give model category structures on the category $\mathcal{S} S p_{\mathcal{O}}$ of Segal precategories with a fixed object set. As in the case of homotopy $\mathcal{T}$-algebras, there is no model structure on the category of Segal categories, due to a lack of colimits.

Proposition 3.13 ([7, 3.7]). There is a model category structure on $\mathcal{S} S p_{\mathcal{O}}$, which we denote $\mathcal{S} S p_{\mathcal{O}, f}$, in which the weak equivalences are levelwise weak equivalences of simplicial sets, the fibrations are the levelwise fibrations of simplicial sets, and the cofibrations are the maps with the left lifting property with respect to the maps which are fibrations and weak equivalences.

Now, we would like to find a map $\varphi_{\mathcal{O}}$ with which to localize $\mathcal{S} \mathcal{S} p_{\mathcal{O}, f}$ so that the fibrant objects are Segal categories. We first consider the map $\varphi$ used by Rezk $[\mathbf{2 1}$, $\S 4]$ to localize simplicial spaces to obtain more general Segal spaces; we then modify it so that the objects are in $\mathcal{S} S p_{\mathcal{O}, f}$. (Rezk's Segal spaces satisfy the same product condition as Segal categories. While they are not necessarily discrete in degree zero, they have the additional requirement that they be Reedy fibrant.)

Using the maps $\alpha^{k}$ given above, Rezk defines the object

$$
G(n)^{t}=\bigcup_{k=0}^{n-1} \alpha^{k} \Delta[1]^{t}
$$

and the inclusion map $\varphi^{n}: G(n)^{t} \rightarrow \Delta[n]^{t}$. His localization is with respect to the coproduct of inclusion maps

$$
\varphi=\coprod_{n \geqslant 1}\left(G(n)^{t} \rightarrow \Delta[n]^{t}\right) .
$$

However, in our case, the objects $G(n)^{t}$ and $\Delta[n]^{t}$ are not necessarily in the category $\mathcal{S} S p_{\mathcal{O}}$. To modify them, we can replace each $\Delta[n]^{t}$ with the objects $\Delta[n]_{\underline{x}}^{t}$, where $\underline{x}=\left(x_{0}, \ldots, x_{n}\right)$ specifies the 0 -simplices. Then, we define

$$
G(n)_{\underline{x}}^{t}=\bigcup_{k=0}^{n-1} \alpha^{k} \Delta[1]_{x_{k}, x_{k+1}}^{t} .
$$

Now, we need to take coproducts not only over all values of $n$, but also over all $n$-tuples of vertices.

So, we define for each $n \geqslant 0$ the map

$$
\varphi^{n}=\coprod_{\underline{x} \in \mathcal{O}^{n+1}}\left(G(n)_{\underline{x}}^{t} \rightarrow \Delta[n]_{\underline{x}}^{t}\right) .
$$


Then the map $\varphi$ looks like

$$
\varphi=\coprod_{n \geqslant 1}\left(\varphi^{n}: \coprod_{\underline{x} \in \mathcal{O}^{n+1}}\left(G(n)_{\underline{x}}^{t} \rightarrow \Delta[n]_{\underline{x}}^{t}\right)\right) .
$$

When the set $\mathcal{O}$ is not clear from the context, we will write $\varphi_{\mathcal{O}}$ to specify that we are in $\mathcal{S} \mathcal{S}_{p_{\mathcal{O}}}$.

For any simplicial space $X$ there is a map

$$
\varphi_{n}=\operatorname{Map}^{h}\left(\varphi^{n}, X\right): \operatorname{Map}^{h}\left(\coprod_{\underline{x}} \Delta[n]_{\underline{x}}^{t}, X\right) \rightarrow \operatorname{Map}^{h}\left(\coprod_{\underline{x}} G(n)_{\underline{x}}^{t}, X\right) .
$$

More simply written, this map is

$$
\varphi_{n}: X_{k} \rightarrow \underbrace{X_{1} \times_{X_{0}} \cdots \times_{X_{0}} X_{1}}_{n}
$$

and is precisely the Segal map given in the definition of a Segal category. Thus, the map $\varphi_{\mathcal{O}}$ is the correct one to use for localizing the model category $\mathcal{S} S p_{\mathcal{O}, f}$.

Proposition 3.14 ([7, 3.8]). Localizing the model category structure on $\mathcal{S} \mathcal{S}_{\mathcal{O}_{\mathcal{O}, f}}$ with respect to the map $\varphi_{\mathcal{O}}$ results in a model category structure $\mathcal{L} \mathcal{S} S p_{\mathcal{O}, f}$ on simplicial spaces with a fixed set $\mathcal{O}$ in degree zero in which the weak equivalences are the $\varphi_{\mathcal{O}}$-local equivalences, the cofibrations are those of $\mathcal{S} \mathcal{S} p_{\mathcal{O}, f}$, and the fibrations are the maps with the right lifting property with respect to the cofibrations which are $\varphi_{\mathcal{O}}$-local equivalences.

We will find it convenient to make our calculations in another model structure with the same weak equivalences but in which all objects are cofibrant.

Theorem $3.15([7,3.9])$. There is a model category structure $\mathcal{S} \mathcal{S}_{\mathcal{O}_{, c}}$ on the category of Segal precategories with a fixed set $\mathcal{O}$ in degree zero in which the weak equivalences and cofibrations are levelwise, and in which the fibrations are the maps with the right lifting property with respect to the acyclic cofibrations. This model structure can then be localized with respect to the map $\varphi_{\mathcal{O}}$ to obtain a model structure which we denote $\mathcal{L S S} p_{\mathcal{O}, c}$.

These two model category structures are in fact equivalent to one another.

Proposition 3.16 ([7, 3.10]). The adjoint pair given by the identity functor induces a Quillen equivalence of model categories

$$
\mathcal{L S S} p_{\mathcal{O}, f} \longleftrightarrow \mathcal{L S S} p_{\mathcal{O}, c} .
$$

Let $*$ denote the set with a single element. The main result of $[\mathbf{7}]$ is that we have the following chain of Quillen equivalences, in which the top-most maps are the left adjoints, which can be composed to form a single Quillen equivalence:

$$
\mathcal{L} \mathcal{S} S p_{*, f} \rightleftarrows \mathcal{L} \mathcal{S} S e t s_{*}^{\mathcal{T}} \rightleftarrows \mathcal{A l g}{ }^{\mathcal{T}_{M}} .
$$




\section{A model for simplicial groups}

We would like to extend the results of $[7]$ to the case of simplicial groups rather than simplicial monoids. In this section, we describe the modifications that need to be made in order to encode the necessary inverses.

In the case of monoids, we consider functors $\boldsymbol{\Delta}^{o p} \rightarrow \mathcal{S} \mathcal{S} e t s$, where the category $\boldsymbol{\Delta}^{o p}$ has as objects finite ordered sets $[n]=(0 \rightarrow 1 \rightarrow \cdots \rightarrow n)$ for each $n \geqslant 0$ and as morphisms the opposites of the order-preserving maps between them. Notice that each $[n]$ can be regarded as a category with $n+1$ objects and a single morphism $i \rightarrow j$ whenever $i \leqslant j$.

Here, we consider instead a category, which we denote $\mathbf{I} \boldsymbol{\Delta}^{o p}$, whose objects are given by small groupoids $I[n]=(0 \rightleftarrows 1 \leftrightarrows \cdots \rightleftarrows n)$ for $n \geqslant 0$. In other words, each $I[n]$ is a category with $n+1$ objects and a single isomorphism between any two objects. The morphisms of $\mathbf{I} \boldsymbol{\Delta}^{o p}$ are generated by two sets of maps: first, the opposite of the order-preserving maps, as we have in $\boldsymbol{\Delta}^{o p}$, and also by a "flip" morphism on each $I[n]$ which sends each $i$ to $n-i$. Note that in this case "order-preserving" should be taken to mean in the standard numerical ordering of the objects of each $I[n]$, even though $I[n]$ cannot be considered to be "ordered" by its morphisms in the same sense that $[n]$ is. Alternatively, thinking of $\mathbf{I} \boldsymbol{\Delta}^{o p}$ as a subcategory of the category of the category of all small groupoids, one should be aware that it is certainly not a full subcategory.

To understand these maps better, we consider, for example, maps

$$
I[2]=(0 \leftrightarrows 1 \leftrightarrows 2) \rightarrow(0 \leftrightarrows 1)=I[1]
$$

Because of the flips, we no longer have only order-preserving maps, but we do have a preservation of "betweenness." Thus, if $0 \mapsto 1$ and $2 \mapsto 1$, then it follows that $1 \mapsto 1$ also. In general, if we have a map $I[n] \rightarrow I[m]$ with $0 \leqslant i<j<k \leqslant n$ such that $i \mapsto \ell$ and $k \mapsto \ell$, for some $0 \leqslant \ell \leqslant m$, it follows that $j \mapsto \ell$ also.

In the case of $\boldsymbol{\Delta}$, the simplicial set $\Delta[n]$ is given by the representable functor $\operatorname{Hom}_{\boldsymbol{\Delta}}(-,[n])$. Similarly, we can define a simplicial set $I \Delta[n]$ which is given by the representable functor $\operatorname{Hom}_{\mathbf{I} \Delta}(-, I[n])$. These "invertible $n$-simplices" are the standard building blocks of the spaces we consider here. In particular, every simplex should be regarded as having a corresponding "inverse" simplex, even though this terminology does not make sense in the usual way, since there is no notion of composition in a simplicial set. As with simplicial sets, we can consider the boundary of $I \Delta[n]$, denoted $I \dot{\Delta}[n]$, which consists the simplices of $I \Delta[n]$ of degree less than $n$.

Thus, we can define an invertible simplicial set to be a functor $\mathbf{I} \boldsymbol{\Delta}^{o p} \rightarrow \mathcal{S}$ ets, and, more generally, an invertible simplicial object in a category $\mathcal{C}$ to be a functor $\mathbf{I} \boldsymbol{\Delta}^{o p} \rightarrow \mathcal{C}$. We denote the category of invertible simplicial sets by ISSets. We further consider the case of invertible simplicial spaces, or functors $\mathbf{I} \boldsymbol{\Delta}^{o p} \rightarrow \mathcal{S} \mathcal{S}$ ets. Since there is a forgetful functor $U: I \mathcal{S} \mathcal{S} e t s \rightarrow \mathcal{S S}$ ets (respectively, $U: \mathcal{S} \mathcal{S e t s}^{I \boldsymbol{\Delta}^{o p}} \rightarrow$ $\mathcal{S} \mathcal{S e t} s^{\boldsymbol{\Delta}^{o p}}$ ), we define a map $f$ of invertible simplicial sets (respectively, spaces) to be a weak equivalence if $U(f)$ is a weak equivalence of simplicial sets (respectively, spaces).

In particular, we define a Segal pregroupoid to be an invertible simplicial space $X$ such that the simplicial set $X_{0}$ is discrete. A Segal pregroupoid is reduced if $X_{0}=\Delta[0]$. To define a Segal groupoid, we need an analogue of the map $\varphi$ which we used to define 
Segal categories. However, just as we defined the maps $\alpha_{k}:[n] \rightarrow[1]$ in $\boldsymbol{\Delta}^{o p}$, we can define maps $\beta_{k}: I[n] \rightarrow I[1]$ in $\mathbf{I} \boldsymbol{\Delta}^{o p}$. Thus, for any invertible simplicial space $X$ and $n \geqslant 2$, we can define the map

$$
\xi_{n}: X_{n} \rightarrow \underbrace{X_{1} \times_{X_{0}} \cdots \times_{X_{0}} X_{1}}_{n}
$$

induced by the maps

$$
X\left(\beta_{k}\right): X_{n} \rightarrow X_{1}
$$

Thus, a Segal groupoid is a Segal pregroupoid $X$ such that for each $n \geqslant 2$ the map $\xi_{n}$ is a weak equivalence of invertible simplicial sets.

As we used the map $\varphi_{\mathcal{O}}$ to localize the model structures $\mathcal{S} \mathcal{S} p_{\mathcal{O}, f}$ and $\mathcal{S} \mathcal{S} p_{\mathcal{O}, c}$, we can define an analogous map $\xi_{\mathcal{O}}$ in this situation. To do so, we first define, for any $n \geqslant 2$, the simplicial space $I G(n)_{\mathcal{O}}^{t}$ and $\underline{x} \in \mathcal{O}^{n+1}$ given by

$$
I G(n)_{\mathcal{O}}^{t}=\bigcup_{k=0}^{n-1} \beta^{k} I \Delta[1]_{x_{k}, x_{k+1}}^{t}
$$

from which we get an inclusion

$$
\xi^{k}: \coprod_{\underline{x} \in \mathcal{O}^{n+1}}\left(I G(n)_{\underline{x}}^{t} \hookrightarrow I \Delta[n]_{\underline{x}}^{t}\right) .
$$

Then we have the map

$$
\xi_{\mathcal{O}}=\coprod_{n \geqslant 1}\left(\xi^{n}: \coprod_{\underline{x} \in \mathcal{O}^{n+1}}\left(G(n)_{\underline{x}}^{t} \rightarrow \Delta[n]_{\underline{x}}^{t}\right)\right) .
$$

Proposition 4.1. There is a model category structure $\mathcal{I S S} p_{\mathcal{O}, f}$ on the category of Segal pregroupoids with a fixed set $\mathcal{O}$ in degree zero in which the weak equivalences and fibrations are given levelwise. Similarly, there is a model category structure $\mathcal{I} \mathcal{S} \mathcal{S}_{\mathcal{O}, c}$ on the same underlying category in which the weak equivalences and cofibrations are given levelwise. Furthermore, we can localize each of these model category structures with the map $\xi_{\mathcal{O}}$ to obtain model structures $\mathcal{I} \mathcal{L S S} p_{\mathcal{O}, f}$ and $\mathcal{I} \mathcal{L S S} p_{\mathcal{O}, c}$ whose fibrant objects are Segal groupoids.

Proof. We begin with the model structure $\mathcal{I S S} p_{\mathcal{O}, f}$, whose existence can be proved analogously to that of $\mathcal{S} \mathcal{S} p_{\mathcal{O}, f}[\mathbf{7}, 3.7]$. We first note that, in an abuse of notation, we denote by $K$ the constant invertible simplicial space given by a simplicial set $K$. Furthermore, if $\underline{x}=\left(x_{0}, \ldots, x_{n}\right) \in \mathcal{O}^{n+1}$, we denote by $I \Delta[n]_{\underline{x}}^{t}$ the invertible simplicial space with the constant simplicial set given by $\underline{x}$ in degree zero. Specifying the vertices of an $n$-simplex in this way is necessary since we require all the morphisms to be the identity on the objects.

Now, we are able to define the objects that we need in order to define sets of generating cofibrations and generating acyclic cofibrations. First, define $\left(I P_{m, n}\right)_{\underline{x}}$ to 
be the pushout of the diagram

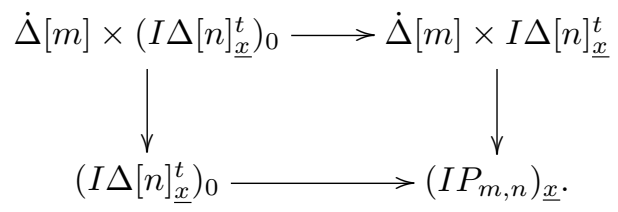

Similarly, define $\left(I Q_{m, n}\right)_{\underline{x}}$ to be the pushout

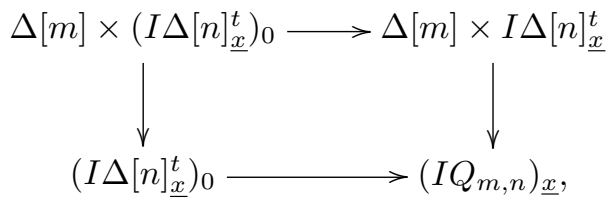

and $\left(I R_{m, n, k}\right)_{\underline{x}}$ to be the pushout

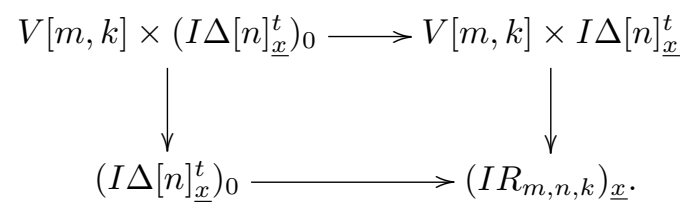

Now, we define the sets

$$
I_{f}=\left\{\left(I P_{m, n}\right)_{\underline{x}} \rightarrow\left(I Q_{m, n}\right)_{\underline{x}} \mid m, n \geqslant 0, \underline{x} \in \mathcal{O}^{n+1}\right\}
$$

and

$$
J_{f}=\left\{\left(I R_{m, n, k}\right)_{\underline{x}} \rightarrow\left(I Q_{m, n}\right)_{\underline{x}} \mid m, n \geqslant 0, k \geqslant 1, \underline{x} \in \mathcal{O}^{n+1}\right\} .
$$

Then, applying Theorem 2.1 to these sets, we can obtain a cofibrantly generated model category structure $\mathcal{I S S} p_{\mathcal{O}, f}$.

Similarly, by defining appropriate sets of maps, we can obtain generating cofibrations and generating acyclic cofibrations for the model structure $\mathcal{I S S} p_{\mathcal{O}, c}$. To define these maps, note that the inclusion functor from the category of Segal pregroupoids to the category of invertible simplicial spaces has a left adjoint which can be called a reduction functor, since it "reduces" the space in degree zero to a discrete space. Given an invertible simplicial space $X$, we denote its reduction by $(X)_{r}$.

Now, we define sets

$$
I_{c}=\left\{\left(\dot{\Delta}[m] \times I \Delta[n]_{\underline{x}}^{t} \cup \Delta[m] \times I \dot{\Delta}[n]_{\underline{x}}^{t}\right)_{r} \rightarrow\left(\Delta[m] \times I \Delta[n]_{\underline{x}}^{t}\right)_{r}\right\}
$$

and

$$
J_{c}=\left\{\left(V[m, k] \times I \Delta[n]_{\underline{x}}^{t} \cup \Delta[m] \times I \dot{\Delta}[n]_{\underline{x}}^{t}\right)_{r} \rightarrow\left(\Delta[m] \times I \Delta[n]_{\underline{x}}^{t}\right)_{r}\right\} .
$$

Again, applying Theorem 2.1, we obtain the model structure $\mathcal{I S S} p_{\mathcal{O}, c}$ just as in $[\mathbf{7}$, 3.9].

Finally, applying Theorem 2.7 with the map $\xi_{\mathcal{O}}$ to each of these model category structures, we can obtain model category structures $\mathcal{I} \mathcal{L S} \mathcal{S}_{p_{\text {O }, f}}$ and $\mathcal{I L S S} p_{\mathcal{O}, c}$.

As in the Segal category case, Proposition 3.16, we have the following result. 
Proposition 4.2. The adjoint pair given by the identity functor induces a Quillen equivalence of model categories

$$
\mathcal{I L S S} p_{\mathcal{O}, f} \longleftrightarrow \mathcal{I} \mathcal{L S S} p_{\mathcal{O}, c} .
$$

We now turn to the theory of groups $\mathcal{T}_{G}$. As with the theory of monoids, we have model structures $\mathcal{A l g}{ }^{\mathcal{T}_{G}}$ and $\mathcal{L S S}$ et $s_{*}^{\mathcal{T}_{G}}$ with Quillen equivalences

$$
\mathcal{A l g}^{\mathcal{T}_{G}} \leftrightarrows \mathcal{L} \mathcal{S} S e t s_{*}^{\mathcal{T}_{G}},
$$

as given in the previous section, where as before $*$ denotes the set with one element. Thus, we need only show that there is a Quillen equivalence

$$
\mathcal{L S S e t s} s_{*}^{\mathcal{T}_{G}} \leftrightarrows \mathcal{I} \mathcal{L S S} p_{*, f}
$$

to prove the following theorem.

Theorem 4.3. The model category structure $\mathcal{A l g}{ }^{\mathcal{T}_{G}}$ is Quillen equivalent to the model category structure $\mathcal{I} \mathcal{L S S} p_{*, f}$.

As in [7], we prove this theorem using several lemmas. Note that in the model structure $\mathcal{I} \mathcal{L S} \mathcal{S} p_{*, c}$, we denote by $L_{1}$ the localization, or fibrant replacement functor. (Again, it is convenient to make our calculations in this category rather than in $\mathcal{I} \mathcal{L S S} p_{*, f}$ because here every object is cofibrant.) Analogously, we denote by $L_{2}$ the localization functor in $\mathcal{L S S e t} s_{*}^{\mathcal{T}_{G}}$. We will make use of the following general result for localizations.

Lemma $4.4([\mathbf{7}, 4.1])$. Let $L$ be a localization functor on a model category $\mathcal{M}$. Given a small diagram of objects $X_{\alpha}$ of $\mathcal{M}$,

$$
L\left(\operatorname{hocolim} X_{\alpha}\right) \simeq L \operatorname{hocolim}\left(L\left(X_{\alpha}\right)\right) .
$$

The first step in the proof of the theorem is to show what the localization functor $L_{1}$ does to the invertible $n$-simplex $I \Delta[n]^{t}$. By Inerve $(-)^{t}$, we denote the invertible nerve functor $\operatorname{Hom}(I[n],-)$.

Proposition 4.5. Let $F_{n}$ denote the free group on $n$ generators. Then in $\mathcal{I} \mathcal{L S} \mathcal{S} p_{*, c}$, $L_{1} I \Delta[n]_{*}^{t}$ is weakly equivalent to Inerve $\left(F_{n}\right)^{t}$ for each $n \geqslant 0$.

Proof. The proof is very similar to the one for reduced Segal categories $[\mathbf{7}, 4.2]$. As in that case, note that when $n=0, I \Delta[0]_{*}^{t}$ is isomorphic to $I$ nerve $\left(F_{0}\right)^{t}$, which is already a Segal groupoid.

Now we consider the case where $n=1$. We want to show that the map

$$
I \Delta[1]_{*}^{t} \rightarrow I \text { nerve }\left(F_{1}\right)^{t}
$$

obtained by localizing with respect to the map $\xi$ is a weak equivalence in $\mathcal{I} \mathcal{L S S} p_{*, c}$.

We first define a filtration $\Psi_{k}$ of $I$ nerve $\left(F_{1}\right)^{t}$ whose set of $j$-simplices looks like

$$
\Psi_{k}\left(\operatorname{Inerve}\left(F_{1}\right)^{t}\right)_{j}=\left\{\left(x^{n_{1}}|\cdots| x^{n_{j}}\right)\left|\sum_{\ell=1}^{j}\right| n_{\ell} \mid \leqslant k\right\},
$$

where $x$ and its "inverse" $x^{-1}$ denote the two nondegenerate 1-simplices of 
$I \Delta[1]_{*}^{t}=\Psi_{1}$. Thus, we have

$$
I \Delta[1]_{*}^{t}=\Psi_{1} \subseteq \Psi_{2} \subseteq \cdots \subseteq \Psi_{k} \subseteq \cdots \subseteq \operatorname{colim}_{k} \Psi_{k} .
$$

We can obtain $\Psi_{2}$ from $\Psi_{1}$ by taking a pushout

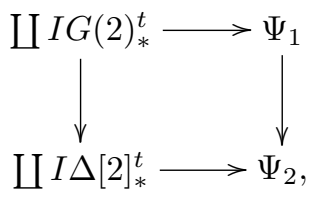

where the coproducts on the left-hand side are taken over all maps $I G(2)_{*}^{t} \rightarrow \Psi_{1}$ and $I G(2)_{*}^{t}$ is as defined at the beginning of the section. This process serves to add in, for example, a "composite" 1 -simplex $x^{2}$ and the 2 -simplex whose boundary consists of 1-simplices $x, x$, and $x^{2}$, as well as the inverses to these new simplices. Notice that since we are working in $\mathcal{I} \mathcal{L S S} p_{*, c}$, the left-hand vertical map is an acyclic cofibration, and therefore $\Psi_{1} \rightarrow \Psi_{2}$ is an acyclic cofibration also [13, 3.14].

Similarly, to obtain $\Psi_{3}$ we will add extra 1-simplices, such as $x^{-3}$ in order to add a 3 -simplex $\left(x^{-1}\left|x^{-1}\right| x^{-1}\right)$. However, when taking the pushout, we do not want to start with $I G(3)_{*}$, since we have already added some of the 1 -simplices of this 3 -simplex when we localized to obtain $\Psi_{2}$. So, we define $\left(I \Delta[3]_{*}^{t}\right)_{\Psi_{2}}$ to be the part of $I \Delta[3]_{*}^{t}$ contained in $\Psi_{2}$. Then we have a pushout diagram

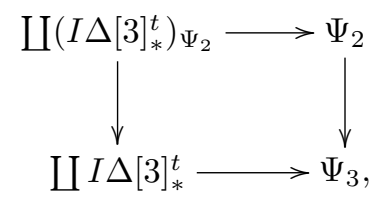

where the coproducts are taken over all maps $\left(I \Delta[3]_{*}^{t}\right)_{\Psi_{2}} \rightarrow \Psi_{2}$. The map $\left(I \Delta[3]_{*}^{t}\right)_{\Psi_{2}} \rightarrow I \Delta[3]_{*}^{t}$ is a weak equivalence in $\mathcal{I} \mathcal{L S S} p_{*, c}$ as follows. Consider the maps

$$
I G(3)_{*}^{t} \stackrel{\alpha}{\longrightarrow}\left(I \Delta[3]_{*}^{t}\right)_{\Psi_{2}} \stackrel{\beta}{\longrightarrow} I \Delta[3]_{*}^{t} .
$$

For any local $X$, the functor $\operatorname{Map}(-, X)$ applied to any of the three above spaces yields $X_{1} \times X_{1} \times X_{1} \simeq X_{3}$. The map $\alpha$ is a weak equivalence since it is just a patching together of two localizations coming from the map $I G(2)_{*}^{t} \rightarrow I \Delta[2]_{*}^{t}$, which is a weak equivalence since it is one of the maps with respect to which we are localizing. The composite map $\beta \alpha$ is also a weak equivalence for the same reason. Thus, $\beta$ is also a weak equivalence by the two-out-of-three property for weak equivalences. Again, since $\left(I \Delta[3]_{*}^{t}\right)_{\Psi_{2}} \rightarrow I \Delta[3]_{*}^{t}$ is an acyclic cofibration in $\mathcal{I} \mathcal{L S S} p_{*, c}$, the map $\Psi_{2} \rightarrow \Psi_{3}$ is an acyclic cofibration also.

For $k>1$, we define $\left(I \Delta[k+1]_{*}^{t}\right)_{\Psi_{k}}$ to be the piece of $I \Delta[k+1]_{*}^{t}$ already obtained from previous steps of the filtration. Note that it always consists of two copies of $I \Delta[k]_{*}^{t}$ attached along a copy of $I \Delta[k-1]_{*}^{t}$, so the same argument as for $k=2$ shows that the map $\left(I \Delta[k+1]_{*}^{t}\right)_{\Psi_{k}} \rightarrow I \Delta[i+1]_{*}^{t}$ is a weak equivalence. Hence, for each $k$ 
we obtain $\Psi_{k+1}$ via the pushout diagram

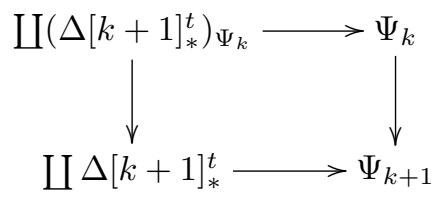

with the coproducts given as before.

Now that we have defined each stage of our filtration, using the bar construction notation shows how to map this local object to $I$ nerve $\left(F_{1}\right)^{t}$. For example,

$$
\left(x\left|x^{-1}\right| x^{2}\right) \mapsto\left(x, x^{-1}, x^{2}\right) \in F_{1} \times F_{1} \times F_{1} .
$$

Using Lemma 4.4 we have that

$$
\begin{aligned}
\operatorname{nerve}\left(F_{1}\right)^{t} & \simeq F_{1}\left(\operatorname{Inerve}\left(F_{1}\right)^{t}\right) \\
& \simeq L_{1}\left(\operatorname{hocolim}\left(\Psi_{k}\right)\right) \\
& \simeq L_{1}\left(\operatorname{hocolim} L_{1}\left(\Psi_{k}\right)\right) \\
& \simeq L_{1}\left(\operatorname{hocolim} L_{1}\left(\Psi_{1}\right)\right) \\
& \simeq L_{1} L_{1}\left(\Psi_{1}\right) \\
& \simeq L_{1}\left(\Psi_{1}\right) \\
& \simeq L_{1}\left(I \Delta[1]_{*}^{t}\right) .
\end{aligned}
$$

Now, for $n=2$ (i.e., starting with $I \Delta[2]_{*}^{t}$ ), we have six 1-simplices, which we call $x, y, x y, x^{-1}, y^{-1}$, and $(x y)^{-1}$, and two nondegenerate 2 -simplices $(x \mid y)$ and $\left(y^{-1} \mid x^{-1}\right)$. Because we now have two variables, we need to define the filtration slightly differently as $\Psi_{i}=\left\{\left[w_{1}, \ldots, w_{k}\right] \mid \ell\left(w_{1} \ldots w_{k}\right) \leqslant i\right\}$, where the $w_{j}$ 's are (unreduced) words in $x$ and $y$ and $\ell$ denotes the length of a given word. Note that by beginning with $\Psi_{1}$ we start with fewer simplices than those of the 2 -simplex we are considering, but by passing to $\Psi_{2}$ we obtain $x y,(x \mid y)$ and their respective "inverses" as well as additional nondegenerate simplices. (In fact, we are actually starting the filtration with $\Psi_{1}=I G(2)_{*}^{t}$.) The localizations proceed as in the case where $n=1$, enabling us to map to $\operatorname{Inerve}\left(F_{2}\right)^{t}$.

For $n \geqslant 3$, the same argument works as for $n=2$, with the filtrations being defined by the lengths of words in $n$ letters. The resulting object is a reduced Segal category weakly equivalent to $I \Delta[n]_{*}^{t}$. Hence, we have that for any $n, L_{1} I \Delta[n]_{*}^{t}$ is weakly equivalent to $I$ nerve $\left(F_{n}\right)^{t}$.

We now define a functor $J: \mathbf{I} \boldsymbol{\Delta}^{o p} \rightarrow \mathcal{T}_{G}$ induced by the invertible nerve construction on a group $G$. For an object $I[n]$ of $\mathbf{I} \boldsymbol{\Delta}$, define $J^{o p}(I[n])=F_{n}$ where $F_{n}$ denotes the free group on $n$ generators, say $x_{1}, \ldots, x_{n}$. In particular, $J^{o p}(I[0])=F_{0}$, the trivial group. Since $G$ has inverses, there is no difficulty in using the category $\mathbf{I} \boldsymbol{\Delta}^{o p}$ rather than $\boldsymbol{\Delta}^{o p}$.

Taking the (invertible) nerve of a simplicial group $G$ results in an invertible simplicial space which at level $k$ looks like

$$
\text { Inerve }(G)_{k}=G^{k}=\operatorname{Hom}_{\mathcal{G} r p}\left(F_{k}, G\right) \text {. }
$$

Thus the invertible simplicial diagram Inerve(-) of representable functors 
$\operatorname{Hom}\left(F_{k},-\right)$ gives rise to an invertible cosimplicial diagram (i.e., a diagram given by $\mathbf{I} \boldsymbol{\Delta})$ of representing objects $T_{k}$.

To obtain an invertible simplicial diagram of free groups, we simply reverse the direction of the arrows to obtain a functor $J: \mathbf{I} \boldsymbol{\Delta}^{o p} \rightarrow \mathcal{T}_{G}$. This map induces a map $J^{*}: \mathcal{S} \operatorname{Sets}^{\mathcal{T}_{G}} \rightarrow \mathcal{S} \mathcal{S} e t s^{\mathbf{I}{ }^{o p}}$, which can be restricted to a map $J^{*}: \mathcal{S} \operatorname{Sets}_{*}^{\mathcal{T}_{G}} \rightarrow \mathcal{I S S} p_{*}$. Notice that $J^{*}$ is the identity on objects but restricts from the morphisms of $\mathcal{T}_{G}$ to those of $\mathbf{I} \boldsymbol{\Delta}^{o p}$.

To obtain a left adjoint to $J^{*}$, we use a left Kan extension. We state the following definitions in a general context. Let $p: \mathcal{C} \rightarrow \mathcal{D}$ and $G: \mathcal{C} \rightarrow \mathcal{S} \mathcal{S}$ ets be functors.

Definition 4.6. If $d$ is an object of $\mathcal{D}$, then the over category or category of objects over $d$, denoted $(p \downarrow d)$, is the category whose objects are pairs $(c, f)$, where $c$ is an object of $\mathcal{C}$ and $f: p(c) \rightarrow d$ is a morphism in $\mathcal{D}$. If $c^{\prime}$ is another object of $\mathcal{C}$, a morphism in the over category is given by a map $c \rightarrow c^{\prime}$ which makes the resulting triangular diagram commute.

Definition $4.7([\mathbf{1 5}, 11.8 .1])$. Let $p, c$, and $G$ be defined as above, and let $f: p(c) \rightarrow d$ be an object in $(p \downarrow d)$. The left Kan extension over $p$ is a functor $p_{*} G: \mathcal{D} \rightarrow \mathcal{S S}$ ets defined by

$$
\left(p_{*} G\right)(d)=\operatorname{colim}_{(p \downarrow d)}((c, f) \mapsto G(c)) .
$$

Note that, since we are making calculations in the model structure $\mathcal{I} \mathcal{L S} \mathcal{S}_{p_{\mathcal{O}, c}}$ in which all objects are cofibrant, taking the left Kan extension is homotopy invariant $[\mathbf{1 2}, 3.7]$.

Proposition $4.8([\mathbf{1 5}, 11.9 .3])$. Let $\mathcal{C} \rightarrow \mathcal{D}$ be a functor. The functor $\mathcal{S} \mathcal{S e t}^{\mathcal{C}} \rightarrow$ $\mathcal{S} \mathcal{S}$ ets ${ }^{\mathcal{D}}$, given by sending $G$ to the left Kan extension $p_{*} G$, is left adjoint to the functor $\mathcal{S} \mathcal{S} e t s^{\mathcal{D}} \rightarrow \mathcal{S} \mathcal{S}$ ets ${ }^{\mathcal{C}}$ given by composition with $p$.

Thus, define $J_{*}: \mathcal{I} \mathcal{S} \mathcal{S} p_{*} \rightarrow \mathcal{S} \mathcal{S}$ ets $s_{*}^{\mathcal{T}_{G}}$ to be the left Kan extension over $J$ which is left adjoint to $J^{*}$. Note that even if $G$ is a reduced Segal groupoid, $J_{*}(G)$ is not necessarily local in $\mathcal{L S S}$ et $s_{*}^{\mathcal{T}_{G}}$. To obtain a $\mathcal{T}_{G}$-algebra, we must apply the localization functor $L_{2}$. So, we want to know what we get when we apply $J_{*}$ followed by $L_{2}$ to a reduced Segal groupoid.

Define $I M[k]$ to be the functor $\mathcal{T}_{G} \rightarrow \mathcal{S} \mathcal{S}$ ets given by $F_{n} \mapsto \operatorname{Hom}_{\mathcal{T}_{G}}\left(F_{k}, F_{n}\right)=F_{k}^{n}$. Let $H$ be the reduced Segal groupoid $I$ nerve $\left(F_{k}\right)^{t}$.

Lemma 4.9. In $\mathcal{L} \mathcal{S} \mathcal{S e t s}_{*}^{\mathcal{T}_{G}}, L_{2} J_{*}(H)$ is weakly equivalent to $I M[k]$.

Proof. The proof is similar to that of $[\mathbf{7}, 4.3]$. It suffices to show that for any local object $X$ in $\mathcal{L S S e t s}{ }_{*}^{\mathcal{T}_{G}}$,

$$
\operatorname{Map}_{\mathcal{L S S e t s}{ }_{*}^{\tau_{G}}}^{h}\left(L_{2} J_{*} H, X\right) \simeq X\left(F_{k}\right)
$$


since $\operatorname{Map}_{\mathcal{L S S e t s}}^{h}{ }^{\tau_{G}}(I M[k], X)$ is precisely $X\left(F_{k}\right)$. This fact can be shown in the following argument:

$$
\begin{aligned}
& \operatorname{Map}_{\mathcal{L S S} \mathcal{S e t s}_{*}^{\tau_{G}}}^{h}\left(L_{2} J_{*} H, X\right) \simeq \operatorname{Map}_{\mathcal{L} \mathcal{S} \mathcal{S e t s}_{*}^{\mathcal{T}_{G}}}^{h}\left(J_{*} H, X\right) \\
& \simeq \operatorname{Map}_{\mathcal{I} \mathcal{L S S} p_{*, c}}^{h}\left(H, J^{*} X\right) \\
& \simeq \operatorname{Map}_{\mathcal{I} \mathcal{L S S} p_{*, c}}^{h}\left(L_{1} I \Delta[k]_{*}^{t}, J^{*} X\right) \\
& \simeq \operatorname{Map}_{\mathcal{I} \mathcal{L S S} p_{*, c}}^{h}\left(I \Delta[k]_{*}^{t}, J^{*} X\right) \\
& \simeq J^{*} X[k] \\
& \simeq X\left(F_{k}\right) \text {. }
\end{aligned}
$$

Proposition 4.10. For any object $X$ in $\mathcal{I S S} p_{*, c}$, we have that $L_{1} X$ is weakly equivalent to $J^{*} L_{2} J_{*} X$.

Proof. This proof is analogous to that of $[\mathbf{7}, 4.7]$. First note that

$$
X \simeq \operatorname{hocolim}_{\mathbf{I} \boldsymbol{\Delta}}{ }^{o p}\left(I[n] \rightarrow \coprod_{i} I \Delta\left[n_{i}\right]^{t}\right),
$$

where the values of $i$ depend on $n$. We begin by looking at $L_{1} X$. Using Lemma 4.4, we have the following:

$$
\begin{aligned}
L_{1} X & \simeq L_{1} \operatorname{hocolim}_{\boldsymbol{\Delta}^{o p}}\left(I[n] \mapsto \coprod I \Delta\left[n_{i}\right]_{*}^{t}\right) \\
& \simeq L_{1} \operatorname{hocolim}_{\mathbf{I} \boldsymbol{\Delta}^{o p}} L_{1}\left(I[n] \mapsto \coprod I \Delta\left[n_{i}\right]_{*}^{t}\right) \\
& \simeq L_{1} \operatorname{hocolim}_{\mathbf{I} \boldsymbol{\Delta}^{o p}}\left(I[n] \mapsto \operatorname{nerve}\left(F_{\sum n_{i}}\right)^{t}\right) .
\end{aligned}
$$

However, hocolim $\boldsymbol{\Delta}^{o p}\left(I[n] \mapsto \operatorname{nerve}\left(F_{\sum n_{i}}\right)^{t}\right)$ is already local, a fact which follows from the fact that the homotopy colimit can be taken at each level, yielding a Segal pregroupoid in $\mathcal{I} \mathcal{L} \mathcal{S} p_{*, c}$ which is still a Segal groupoid.

Working from the other side of the desired equation, we obtain, using the fact that left adjoints commute with homotopy colimits:

$$
\begin{aligned}
J^{*} L_{2} J_{*} X & \simeq J^{*} L_{2} J_{*} \operatorname{hocolim}_{\mathbf{I} \Delta^{o p}}\left(I[n] \mapsto \coprod I \Delta\left[n_{i}\right]_{*}^{t}\right) \\
& \simeq J^{*} L_{2} \operatorname{hocolim}_{\mathbf{I} \Delta^{o p}} J_{*}\left(I[n] \mapsto \coprod I \Delta\left[n_{i}\right]_{*}^{t}\right) \\
& \simeq J^{*} L_{2} \operatorname{hocolim}_{\mathbf{I} \boldsymbol{\Delta}^{o p}} L_{2} J_{*}\left(I[n] \mapsto \coprod I \Delta\left[n_{i}\right]_{*}^{t}\right) \\
& \simeq J^{*} L_{2} \operatorname{hocolim}_{\mathbf{I} \boldsymbol{\Delta} o p}\left(I[n] \mapsto I M\left[\sum n_{i}\right]\right) .
\end{aligned}
$$

At each level, we have the same spaces as in the $\mathbf{I} \boldsymbol{\Delta}^{o p}$-diagram, but with maps given by $\mathcal{T}_{G}$ rather than $\mathbf{I} \boldsymbol{\Delta}^{o p}$. Thus, applying the restriction map $J^{*}$ results in a diagram with the same objects at each level, as we wished to show.

Combining the above results, the following proposition can be proved just as $[7,4.8]$.

Proposition 4.11. The adjoint pair

$$
J_{*}: \mathcal{I S S} p_{*, f} \rightleftarrows \mathcal{S S e t} s_{*}^{\mathcal{T}_{G}}: J^{*}
$$

is a Quillen pair. 
Proof. We first show that the adjoint pair

$$
J_{*}: \mathcal{S S} p_{*, f} \longleftrightarrow \mathcal{S S e t s}{ }_{*}^{\mathcal{T}_{M}}: J^{*}
$$

is a Quillen pair. In both model categories, the fibrations and weak equivalences are defined levelwise. Since the right adjoint $J^{*}$ preserves the spaces at each level, it must preserve both fibrations and acyclic fibrations.

An application of Theorem 2.8 shows that we still have a Quillen pair after the respective localizations.

Proof of Theorem 4.3. First, we need to know that the right adjoint $J^{*}$ reflects weak equivalences between fibrant objects. In each of the two localized model categories $\mathcal{I} \mathcal{S} \mathcal{S} p_{*, f}$ and $\mathcal{L S S e t s} *_{*}^{\mathcal{T}_{G}}$, an object is fibrant if and only if it is local and fibrant in the unlocalized model category. Therefore, in each case a weak equivalence between fibrant objects is a levelwise weak equivalence. Since $J^{*}$ does not change the spaces at each level, it must reflect weak equivalences between fibrant objects.

Finally, by Proposition $4.10, L_{1} X \simeq J^{*} L_{2} J_{*} X$ for any functor $X: \boldsymbol{\Delta}^{o p} \rightarrow \mathcal{S S e t s}$, and in particular for any cofibrant $X$.

Now composing the Quillen equivalences

$$
\mathcal{I} \mathcal{L S S} p_{*, f} \rightleftarrows \mathcal{L S S} \text { ets }_{*}^{\mathcal{T}_{G}} \rightleftarrows \mathcal{A l g}{ }^{\mathcal{T}_{G}}
$$

(where the left adjoint functors are the topmost maps) results in a single Quillen equivalence

$$
\mathcal{I} \mathcal{L S S} p_{*, f} \rightleftarrows \mathcal{A l g}{ }^{\mathcal{T}_{G}}
$$

\section{Segal groupoids}

In this section, we generalize the result on simplicial groups and reduced Segal pregroupoids to one on simplicial groupoids and Segal groupoids. While Segal groupoids (and their $n$-categorical analogues) have been discussed in works by Hirschowitz and Simpson $[\mathbf{1 6}, \mathbf{2 5}]$, we give an alternate but equivalent description of them.

Definition 5.1. A simplicial category is a category enriched over simplicial sets, or a category in which there is a simplicial set of morphisms between any two objects. A simplicial groupoid is a simplicial category enriched over invertible simplicial sets.

For convenience, we describe a modification of the categories $\boldsymbol{\Delta}^{o p}$ and $I \boldsymbol{\Delta}^{o p}$. Let $\mathcal{O}$ be a set. We define the category $\boldsymbol{\Delta}_{\mathcal{O}}^{o p}$ as follows. The objects are given by $[n]_{x_{0}, \ldots, x_{n}}$, where $n \geqslant 0$ and $\left(x_{0}, \ldots x_{n}\right) \in \mathcal{O}^{n+1}$. The $[n]$ should be thought of as in the simplicial category $\boldsymbol{\Delta}^{o p}$; however, recall that when we work with Segal categories we will require all morphisms to preserve the objects. Therefore, we need to have a separate $[n]$ for each possible $(n+1)$-tuple of objects in $\mathcal{O}$. The morphisms in $\boldsymbol{\Delta}_{\mathcal{O}}^{o p}$ are those of $\boldsymbol{\Delta}^{o p}$ but depend on the choice of $\left(x_{0}, \ldots, x_{n}\right)$. Specifically, the face maps are

$$
d_{i}:[n]_{x_{0}, \ldots, x_{n}} \rightarrow[n-1]_{x_{0}, \ldots, \widehat{x}_{i}, \ldots, x_{n}}
$$

and the degeneracy maps are

$$
s_{i}:[n]_{x_{0}, \ldots, x_{n}} \rightarrow[n+1]_{x_{0}, \ldots, x_{i-1}, x_{i}, x_{i}, x_{i+1}, \ldots, x_{n}} .
$$

Note that if $\mathcal{O}$ is the one-object set, then $\boldsymbol{\Delta}_{\mathcal{O}}^{o p}$ is just $\boldsymbol{\Delta}^{o p}$. 
Similarly, we can describe the category $\mathbf{I} \boldsymbol{\Delta}_{\mathcal{O}}^{o p}$. Note that in this case the flip map is no longer a self-map (unless $\mathcal{O}$ consists of a single element), but instead a map $[n]_{x_{0}, \ldots, x_{n}} \rightarrow[n]_{x_{n}, \ldots, x_{0}}$.

Now we can use this notation to describe Segal categories and Segal groupoids. A Segal category with $\mathcal{O}$ in degree zero is a functor $X: \boldsymbol{\Delta}_{\mathcal{O}}^{o p} \rightarrow \mathcal{S S}$ ets such that for each $n \geqslant 2$ and $(n+1)$-tuple $\left(x_{0}, \ldots, x_{n}\right)$, the map

$$
X\left([n]_{x_{0}, \ldots, x_{n}}\right) \rightarrow X\left([1]_{x_{0}, x_{1}}\right) \times_{X[0]_{x_{1}}} \cdots \times_{X[0]_{x_{n-1}}} X\left([1]_{x_{n-1}, x_{n}}\right)
$$

is a weak equivalence. Analogously, a Segal groupoid with $\mathcal{O}$ in degree zero is a functor $X: I \boldsymbol{\Delta}_{\mathcal{O}}^{o p} \rightarrow \mathcal{S} \mathcal{S}$ ets satisfying these same conditions.

Recall from Section 2 that we have model category structures $\mathcal{L} \mathcal{S} \mathcal{S} p_{\mathcal{O}, f}$ and $\mathcal{L} \mathcal{S} S p_{\mathcal{O}, c}$ on the category of Segal precategories with $\mathcal{O}$ in degree zero, in each of which the fibrant objects are Segal categories. Then, from Section 3, we have the analogous model structures $\mathcal{I} \mathcal{L S S} p_{\mathcal{O}, f}$ and $\mathcal{I} \mathcal{L S} \mathcal{S}_{p_{\mathcal{O}, c}}$, whose fibrant objects are Segal groupoids.

We would like to think of the category of simplicial categories, or the category of simplicial groupoids, with object set $\mathcal{O}$ as a diagram category as well. To do so, we need to define the notion of a multi-sorted algebraic theory. To see more details, see $[6]$.

Definition 5.2. Given a set $S$, an $S$-sorted algebraic theory (or multi-sorted theory) $\mathcal{T}$ is a small category with objects $T_{\underline{\alpha}^{n}}$ where $\underline{\alpha}^{n}=\left\langle\alpha_{1}, \ldots, \alpha_{n}>\right.$ for $\alpha_{i} \in S$ and $n \geqslant 0$ varying, and such that each $T_{\underline{\alpha}^{n}}$ is equipped with an isomorphism

$$
T_{\underline{\alpha}^{n}} \cong \prod_{i=1}^{n} T_{\alpha_{i}} .
$$

For a particular $\underline{\alpha}^{n}$, the entries $\alpha_{i}$ can repeat, but they are not ordered. There exists a terminal object $T_{0}$ (corresponding to the empty object of $S$ ).

In particular, we can talk about the theory of $\mathcal{O}$-categories, which we will denote by $\mathcal{T}_{\mathcal{O C} a t}$, and the theory of $\mathcal{O}$-groupoids, which we denote $\mathcal{T}_{\mathcal{O G} d}$. To define these theories, first consider the category $\mathcal{O C}$ at whose objects are the categories with a fixed object set $\mathcal{O}$ and whose morphisms are the functors which are the identity map on the objects. The objects of $\mathcal{T}_{\mathcal{O C} \text { at }}$ are categories which are freely generated by directed graphs with vertices corresponding to the elements of the set $\mathcal{O}$. This theory is sorted by pairs of elements in $\mathcal{O}$, corresponding to the morphisms with source the first element and target the second. In other words, this theory is $(\mathcal{O} \times \mathcal{O})$-sorted $[\mathbf{6}, 3.5]$. (In the one-object case, we get the ordinary theory of monoids, since a monoid is just a category with one object.) We can then say that a simplicial category with object set $\mathcal{O}$ is essentially a strict $\mathcal{T}_{\mathcal{O C} a t}$-algebra, where the definitions of strict and homotopy $\mathcal{T}$-algebras for multi-sorted theories $\mathcal{T}$ are defined analogously to those for ordinary algebraic theories. The objects of $\mathcal{T}_{\mathcal{O G} d}$ are representatives of the isomorphism classes of finitely generated free groupoids with object set $\mathcal{O}$, and a simplicial groupoid is essentially a strict $\mathcal{T}_{\mathcal{O G}} d^{-}$-algebra.

Again, we have a model structure $\mathcal{A} l g^{\mathcal{T}}$ on the category of all $\mathcal{T}$-algebras and a model structure $\mathcal{S} \mathcal{S} e s^{\mathcal{T}}$ on the category of all functors $\mathcal{T} \rightarrow \mathcal{S} \mathcal{S}$ ets, which can be localized as before to obtain a model category structure $\mathcal{L S} \mathcal{S}$ ets ${ }^{\mathcal{T}}$ in which the 
local objects are homotopy $\mathcal{T}$-algebras $[\mathbf{6}, 4.11]$. For $\mathcal{T}_{\mathcal{O C} a t}$, we can define a category $\mathcal{S} \mathcal{S} e t s_{\mathcal{O}}^{\mathcal{T}_{\mathcal{O C} a t}}$ of functors $\mathcal{T}_{\mathcal{O C} a t} \rightarrow \mathcal{S S}$ ets, which send $T_{0}$ to $\amalg_{\mathcal{O}} \Delta[0]$. Making modifications as in the case of $\mathcal{L S S}$ et $s_{*}^{\mathcal{T}_{M}}$, we can define a model structure $\mathcal{L} \mathcal{S} \mathcal{S}$ et $s_{\mathcal{O}}^{\mathcal{T}_{\mathcal{O}} \text { at }}$

which is Quillen equivalent to $\mathcal{A l g}^{\mathcal{T}_{\mathcal{O C a t}}}[\mathbf{8}]$. Similarly, we can define $\mathcal{L} \mathcal{S} \mathcal{S e t s}_{\mathcal{O}^{\mathcal{T}_{\mathcal{G}} d}}$ and show that it is Quillen equivalent to $\mathcal{A l g}^{\mathcal{T}_{\mathcal{O} G d}}$.

In particular, Theorem 3.8 for algebraic theories can be generalized to the case of multi-sorted theories, and therefore we have that there is a Quillen equivalence of model categories between $\mathcal{A l g}^{\mathcal{T}}$ and $\mathcal{L S S}$ ets ${ }^{\mathcal{T}}$ for any multi-sorted theory $\mathcal{T}$ [6, 5.1]. We can again use the version with the stricter requirement on degree zero to obtain a Quillen equivalence

$$
\mathcal{L S S e t s} s_{\mathcal{O}}^{\mathcal{T}_{\mathcal{O} a t}} \rightleftarrows \mathcal{A l g}{ }^{\mathcal{T}_{\mathcal{O C} a t}} .
$$

Hence, the problem reduces to finding a Quillen equivalence between $\mathcal{L} \mathcal{S} \mathcal{S}_{\mathcal{O}, f}$ and $\mathcal{L} \mathcal{S} \operatorname{Sets}_{\mathcal{O}}^{\mathcal{T}_{\text {OCat }}}$.

Theorem $5.3([7,5.5])$. The adjoint pair

$$
J_{*}: \mathcal{L S S} p_{\mathcal{O}, f} \longleftrightarrow \mathcal{L S S e t} s_{\mathcal{O}}^{\mathcal{T}_{\mathcal{O C} a t}}: J^{*}
$$

is a Quillen equivalence.

We can extend this result just as we did in the previous section to obtain a result relating Segal groupoids with object set $\mathcal{O}$ to homotopy algebras over a multi-sorted theory of groupoids with object set $\mathcal{O}$.

The same methods can be used to prove the following.

Theorem 5.4. There exists an adjoint pair

$$
J_{*}: \mathcal{I} \mathcal{L S S} p_{\mathcal{O}, f} \longleftrightarrow \mathcal{L S S e t}_{\mathcal{O}^{\mathcal{T}_{\mathcal{O} d}}}: J^{*}
$$

which is a Quillen equivalence.

Hence, composing Quillen equivalences results in a Quillen equivalence

$$
\mathcal{A l g}{ }^{\mathcal{T}_{G d}} \leftrightarrows \mathcal{I} \mathcal{L S S} p_{\mathcal{O}, f}
$$

The more general result that there is a Quillen equivalence between model structures on the category of all Segal precategories and the category of all small simplicial categories $[\mathbf{9}, 8.6]$ can be extended to the groupoid situation, but we defer its proof to the next paper [5].

\section{An alternative model for simplicial groups}

In this section, we summarize a result of Bousfield for modelling simplicial groups with $\boldsymbol{\Delta}$ by changing the projection maps and therefore the Segal maps [11]. This approach is more convenient for adaptation to the case of abelian groups, as we show in the next section.

Let us recall the way we used $\boldsymbol{\Delta}$ to obtain a model for simplicial monoids. Given a reduced Segal precategory $X: \boldsymbol{\Delta}^{o p} \rightarrow \mathcal{S}$ Sets, the Segal map $X_{n} \rightarrow\left(X_{1}\right)^{n}$ was induced by $\alpha^{k}:[1] \rightarrow[n]$ in $\boldsymbol{\Delta}$ for each $0 \leqslant k \leqslant n-1$, where $\alpha^{k}(0)=k$ and $\alpha^{k}(1)=k+1$. 
Heuristically, we are thinking of the $\left(X_{1}\right)^{n}$ as a chain of $k$ morphisms which has a "composite" (at least up to homotopy) if the Segal map is to be a weak equivalence.

To consider a basic example, consider a 2 -simplex with vertices $\{a, b, c\}$ and 1-simplices $\{a \rightarrow b, b \rightarrow c, a \rightarrow c\}$. The 2-chain here is $a \rightarrow b \rightarrow c$, the composite is given by $a \rightarrow c$, and the 2 -simplex is defined by all three. The first projection map sends the 2 -simplex to $a \rightarrow b$ and the second projection sends it to $b \rightarrow c$.

The idea behind Bousfield's construction is to define these projections differently. In the situation just described, the first projection remains the same. The second projection, however, sends the 2 -simplex to $a \rightarrow c$. Thus, if we are going to fill in the third 1-simplex, we get the "inverse" of the first projection composed with the second projection.

To formalize this construction, we define in $\boldsymbol{\Delta}$ the maps $\gamma^{k}:[1] \rightarrow[n]$ given by $0 \mapsto 0$ and $1 \mapsto k+1$ for all $0 \leqslant k<n$. Again restricting to Segal precategories, we can consider the Bousfield-Segal map $\psi_{n}: X_{n} \rightarrow\left(X_{1}\right)^{n}$ induced by these maps. The models for simplicial groups in this sense will be the reduced Segal categories for which the Bousfield-Segal maps are weak equivalences of simplicial sets for all $n \geqslant 2$. We call such simplicial spaces reduced Bousfield-Segal categories.

To give a localized model structure, we define for each $k \geqslant 2$ the simplicial space

$$
H(k)_{*}^{t}=\bigcup_{i=1}^{k-1} \gamma^{i} \Delta[1]_{*}^{t} \subseteq \Delta[k]_{*}^{t} .
$$

Then, as in the previous situations, define the map

$$
\psi_{*}=\coprod_{k \geqslant 1}\left(\psi^{k}: H(k)_{*}^{t} \rightarrow \Delta[k]_{*}^{t}\right) .
$$

Proposition 6.1. Localizing the model category structure $\mathcal{S} S p_{*, f}$ with respect to the map $\psi_{*}$ results in a model category structure $\mathcal{L}_{B} \mathcal{S} \mathcal{S} p_{*, f}$ whose fibrant objects are reduced Bousfield-Segal categories. There is also an analogous model structure $\mathcal{L}_{B} \mathcal{S} \mathcal{S} p_{*, c}$.

We denote by $L_{B}$ the localization functor in $\mathcal{L}_{B} \mathcal{S} \mathcal{S}_{p_{\mathcal{O}, c}}$. With some minor technical changes, our proof that Segal categories model simplicial monoids generalizes to show that Bousfield-Segal categories model simplicial groups. As with the previous proof, the key point is the analogue of Lemma 4.5 .

Proposition 6.2. Let $F_{n}$ denote the free group on $n$ generators. Then in $\mathcal{L}_{B} \mathcal{S} \mathcal{S}_{\mathrm{O}_{,}, c}$, $L_{B} \Delta[n]_{*}^{t}$ is weakly equivalent to nerve $\left(F_{n}\right)^{t}$.

Proof. In the case where $n=0$, we have that $\Delta[0]_{*}^{t} \cong$ nerve $\left(F_{0}\right)^{t}$. So, we consider the case where $n=1$. As before, we define a filtration $\Psi_{1} \subseteq \Psi_{2} \subseteq \cdots \subseteq \Psi_{k} \subseteq \cdots$. We will use a bar construction notation as before, but, as we are assuming each slot is given by the image of a projection map, its meaning has changed as we have changed our projection maps. The set of $j$-simplices (for $j>1$ ) of $\Psi_{k}$ is given by

$$
\Psi_{k}\left(\operatorname{nerve}\left(F_{1}\right)^{t}\right)_{j}=\left\{\left(x^{n_{1}}|\cdots| x^{n_{j}}\right)\right\}
$$


with the following additional conditions on the superscripts $n_{\ell}$. As before, we require

$$
\sum_{\ell=1}^{j}\left|n_{\ell}\right| \leqslant k
$$

Furthermore, the construction imposes the following conditions depending on $k$. For $k=1,2$, we have $0 \leqslant n_{\ell} \leqslant 1$. For $k=3$, we have $-1 \leqslant n_{\ell} \leqslant 1$. For all $k \geqslant 4$, we have $-k+1 \leqslant n_{\ell} \leqslant k-2$. Note that our definition of $\Psi_{1}$ coincides with that of $\Delta[1]_{*}^{t}$ since each has only one non-degenerate 1 -simplex $x$ and no nondegenerate 2 -simplices. Thus we have

$$
\Delta[1]_{*}^{t}=\Psi_{1} \subseteq \Psi_{2} \subseteq \cdots \subseteq \Psi_{k} \subseteq \cdots \operatorname{colim}_{m} \Psi_{m} .
$$

Setting $k=2$, we can take a pushout

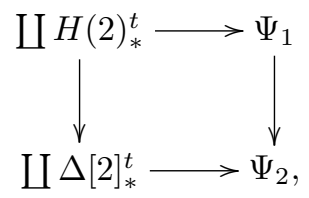

where the coproducts on the left-hand side are over the maps $H(2)_{*}^{t} \rightarrow \Psi_{1}$. In doing so, we obtain the 1 -simplex $x^{-1}$ and 2 -simplices given by $(1 \mid 1),(1 \mid x),(x \mid 1)$, and $(x \mid x)$, where the third is the one which requires $x^{-1}$. (Note that our use of the bar construction notation has been adapted to our new projections.)

Then for $k \geqslant 3, \Psi_{k}$ is obtained from $\Psi_{k-1}$ by taking pushouts along the maps $\amalg\left(\Delta[k]_{*}^{t}\right)_{\Psi_{k-1}} \rightarrow \coprod \Delta[k]_{*}^{t}$ for each $k \geqslant 3$, where $\left(\Delta[k]_{*}^{t}\right)_{\Psi_{k-1}}$ denotes the piece of $\Delta^{t}[k]_{*}$ which we have already obtained. As usual, the coproduct is taken over all maps $\left(\Delta[k]_{*}^{t}\right)_{\Psi_{k-1}} \rightarrow \Psi_{k-1}$.

Then, we can use the inclusions

$$
H(k)_{*}^{t} \rightarrow\left(\Delta[k]_{*}^{t}\right)_{\Psi_{k-1}} \rightarrow \Delta[k]_{*}^{t}
$$

to prove the fact that each map $\Psi_{k-1} \rightarrow \Psi_{k}$ is a weak equivalence, as in the proof of Lemma 4.5 or that of $[\mathbf{7}, 4.2]$.

The rest of the proof follows similarly to the one in Section 4. As in Section 5, it can also be generalized to obtain a result for more general Segal groupoids. We should note that Bousfield's result was stated in terms of homotopy categories and that his proof did not make use of model categories. Furthermore, he worked in more generality, finding a model for $n$-fold loop spaces.

\section{A model for simplicial abelian groups}

In [24], Segal defines $\Gamma$-spaces and shows that strict $\Gamma$-spaces are equivalent to simplicial abelian monoids. Here, we begin with his definition of the category $\Gamma$. Its objects are representatives of isomorphism classes of finite sets, and a morphism $S \rightarrow T$ is given by a map $\theta: S \rightarrow \mathcal{P}(T)$ such that $\theta(\alpha)$ and $\theta(\beta)$ are disjoint whenever $\alpha \neq \beta$. (We denote by $\mathcal{P}(T)$ the power set, or set of all subsets of the set $T$.)

We can then define the opposite category $\Gamma^{o p}$, which has the following description of its own. It is the category with objects $\mathbf{n}=\{0,1, \ldots, n\}$ for $n \geqslant 0$ and morphisms 
$\mathbf{m} \rightarrow \mathbf{n}$ such that $0 \mapsto 0$. Here, we find it convenient to use both descriptions, depending on the situation.

Segal defines a $\Gamma$-space $X$ to be a functor $\Gamma \rightarrow \mathcal{S S}$ ets such that $X_{0} \simeq \Delta[0]$ and the Segal map $\varphi_{k}: X_{k} \rightarrow\left(X_{1}\right)^{k}$ is a weak equivalence of simplicial sets. He further mentions that if $X_{0}=\Delta[0]$ and if each Segal map $\varphi_{k}: X_{k} \rightarrow\left(X_{1}\right)^{k}$ is an isomorphism rather than a weak equivalence, i.e., if $X$ is a strict $\Gamma$-space, then $X$ is essentially a simplicial abelian monoid. (The following proof sketch is due to Badzioch.)

Proposition 7.1 ([24]). The category of strict $\Gamma$-spaces is equivalent to the category of simplicial abelian monoids.

Sketch of proof. First, recall that the category of simplicial abelian monoids is equivalent the the category $\mathcal{A} l g^{\mathcal{T}_{A M}}$, where $\mathcal{T}_{A M}$ is the theory of abelian monoids.

Among the maps of $\Gamma^{o p}$, there are projections $p_{n, i}: \mathbf{n} \rightarrow \mathbf{1}$, where $p_{n, i}(k)=1$ if $k=i$ and 0 otherwise. Then there exists a projection-preserving functor $\Gamma^{o p} \rightarrow \mathcal{T}_{A M}$, where $\mathcal{T}_{A M}$ denotes the theory of abelian monoids. Given a strict $\Gamma$-space $X$, it is uniquely determined by each $X_{n}$, the projection maps $X_{n} \rightarrow X_{1}$, and the map $X_{2} \rightarrow X_{1}$ which is the image of the map $\mathbf{2} \rightarrow \mathbf{1}$ given by $0 \mapsto 0$ and $1,2 \mapsto 1$. In particular, this map $X_{2} \rightarrow X_{1}$ induces (by induction) all maps $X_{n} \rightarrow X_{1}$ arising from the morphisms $\mathbf{n} \rightarrow \mathbf{1}$ given by $0 \mapsto 0$ and $i \mapsto 1$ for all $0<i \leqslant n$.

Then, a strict $\Gamma$-space gives the space $X_{1}$ the structure of an abelian monoid with multiplication map given by the specified map $X_{2} \rightarrow X_{1}$. In particular, $X_{1}$ defines a $\mathcal{T}_{A M}$-algebra $t X$ which is given by the necessary $X_{n}$ for each $n \geqslant 2$ and the projection maps. Then, notice that restricting the $\mathcal{T}_{A M}$-algebra $t X$ to $\Gamma^{o p}$ results in our original $X$. In other words, if $F$ is the forgetful functor from the category of $\mathcal{T}_{A M}$-algebras to the category of strict $\Gamma^{o p}$-spaces, we have that $t F(X)=X$. Thus, the functors $t$ and $X$ are inverse to one another.

Segal defines a functor $\boldsymbol{\Delta} \rightarrow \Gamma$ as follows. The object $[n]$ is sent to $\mathbf{n}$ for each $n \geqslant 0$, and a map $f:[m] \rightarrow[n]$ is sent to the map $\theta: \mathbf{m} \rightarrow \mathbf{n}$ given by $\theta(i)=\{j \in \mathbf{n}$ $f(i-1)<j \leqslant f(i)\}$. In particular, the maps $\alpha^{k}:[1] \rightarrow[n]$ are sent to maps $\theta: \mathbf{1} \rightarrow \mathbf{k}$ given by $\theta(1)=\{k+1\}$.

Now, we would like to know what happens if we define the projections as Bousfield does. Given $\gamma^{k}:[1] \rightarrow[n]$ in $\boldsymbol{\Delta}$, it is sent to the map $j^{k}: \mathbf{1} \rightarrow \mathbf{n}$ given by $j^{k}(1)=$ $\{1, \ldots, k+1\}$. Thus, we can use the maps $j_{k}: \mathbf{1} \rightarrow \mathbf{n}$ to define a modified version of the conditions for a $\Gamma$-space.

Now, a strict Bousfield $\Gamma$-space is a functor $X: \Gamma^{o p} \rightarrow \mathcal{S}$ Sets such that $X(\mathbf{0})=$ $\Delta[0]$ and the maps $X(\mathbf{n}) \rightarrow X(\mathbf{1})^{n}$ induced by the maps $j_{k}$ for all $1 \leqslant k \leqslant n$ are isomorphisms for all $n \geqslant 2$. Similarly, a (homotopy) Bousfield $\Gamma$-space has $X(\mathbf{0})$ contractible and the above map a weak equivalence of simplicial sets. The above proof for strict $\Gamma$-spaces extends to this new situation.

Theorem 7.2. There is an equivalence between the category of strict Bousfield $\Gamma$ spaces and the category of simplicial abelian groups.

However, it is expected, in analogy with the original work of Segal [24], that the (homotopy) Bousfield $\Gamma$-spaces are not equivalent to simplicial abelian groups up to homotopy. Since Segal shows that $\Gamma$-spaces are equivalent to infinite loop spaces when 
they have homotopy inverses $[\mathbf{2 4}, 1.4]$, it is expected that the Bousfield $\Gamma$-spaces are equivalent to infinite loop spaces. Another interesting comparision to be made is with the work of Schwänzl and Vogt [22].

\section{References}

[1] B. Badzioch, Algebraic theories in homotopy theory, Ann. of Math. (2) 155 (2002), no. 3, 895-913.

[2] B. Badzioch, K. Chung, and A.A. Voronov, The canonical delooping machine, J. Pure Appl. Algebra 208 (2007), no. 2, 531-540.

[3] C. Barwick, private communication.

[4] C. Berger, Iterated wreath product of the simplex category and iterated loop spaces, Adv. Math. 213 (2007), no. 1, 230-270.

[5] J.E. Bergner, Adding inverses to diagrams II: Invertible homotopy theories are spaces, Homology, Homotopy and Applications 10 (2008), no. 2, 175-193.

[6] J.E. Bergner, Rigidification of algebras over multi-sorted theories, Algebr. Geom. Topol. 6 (2006), 1925-1955.

[7] J.E. Bergner, Simplicial monoids and Segal categories, Contemp. Math. 431 (2007), 59-83.

[8] J.E. Bergner, Correction to "Simplicial monoids and Segal categories", available at math. AT/0806.1767.

[9] J.E. Bergner, Three models for the homotopy theory of homotopy theories, Topology 46 (2007), 397-436.

[10] F. Borceux, Handbook of Categorical Algebra, Volume 2, Encyclopedia of Mathematics and its Applications 51, Cambridge University Press, Cambridge, 1994.

[11] A.K. Bousfield, The simplicial homotopy theory of iterated loop spaces, unpublished manuscript.

[12] W.G. Dwyer and D.M. Kan, Equivalences between homotopy theories of diagrams, Algebraic topology and algebraic K-theory (Princeton, N.J., 1983), 180205, Ann. of Math. Stud. 113, Princeton Univ. Press, Princeton, NJ, 1987.

[13] W.G. Dwyer and J. Spalinski, Homotopy theories and model categories, in Handbook of Algebraic Topology, 73-126, North-Holland, Amsterdam, 1995.

[14] P.G. Goerss and J.F. Jardine, Simplicial Homotopy Theory, Progress in Math. 174, Birkhäuser Verlag, Basel, 1999.

[15] P.S. Hirschhorn, Model Categories and Their Localizations, Mathematical Surveys and Monographs 99, American Mathematical Society, Providence, RI, 2003.

[16] A. Hirschowitz and C. Simpson, Descente pour les $n$-champs, preprint available at math. AG/9807049.

[17] M. Hovey, Model Categories, Mathematical Surveys and Monographs 63, American Mathematical Society, Providence, RI, 1999.

[18] F.W. Lawvere, Functorial semantics of algebraic theories, Proc. Nat. Acad. Sci. U.S.A. 50 (1963), 869-872. 
[19] S. MacLane, Categories for the Working Mathematician, Second Edition, Graduate Texts in Mathematics 5, Springer-Verlag, New York, 1998.

[20] D. Quillen, Homotopical Algebra, Lecture Notes in Math. 43, Springer-Verlag, New York, 1967.

[21] C. Rezk, A model for the homotopy theory of homotopy theory, Trans. Amer. Math. Soc. 353 (2001), no. 3, 973-1007.

[22] R. Schwänzl and R.M. Vogt, $E_{\infty}$-monoids with coherent homotopy inverses are abelian groups, Topology 28 (1989), no. 4, 481-484.

[23] S. Schwede, Stable homotopy of algebraic theories, Topology 40 (2001), 1-41.

[24] G. Segal, Categories and cohomology theories, Topology 13 (1974), 293-312.

[25] C. Simpson, Effective generalized Seifert-Van Kampen: how to calculate $\Omega X$, preprint available at $\mathrm{q}-\mathrm{alg} / 9710011$.

[26] J.D. Stasheff, Homotopy associativity of $H$-spaces I, Trans. Amer. Math. Soc. 108 (1963), 275-292.

[27] R.W. Thomason, Uniqueness of delooping machines, Duke Math. J. 46 (1979), no. 2, 217-252.

Julia E. Bergner bergnerj@member .ams .org

Department of Mathematics, University of California, Riverside, CA 92521, USA

Previous address: Department of Mathematics, Kansas State University, 138 Cardwell Hall, Manhattan, KS 66506, USA 